\title{
Sex hormones adjust "sex-specific" reactive and diurnal cortisol profiles
}

\author{
Robert-Paul Juster $^{\mathrm{a}, \mathrm{b}, \mathrm{c}}$, Catherine Raymond ${ }^{\mathrm{a}, \mathrm{b}, \mathrm{d}}$, Alexandra Bisson Desrochers ${ }^{\mathrm{a}, \mathrm{e}, \mathrm{f}}$, \\ Olivier Bourdon $^{\mathrm{a}}$, Nadia Durand ${ }^{\mathrm{a}, \mathrm{b}}$, Nathalie Wan ${ }^{\mathrm{a}, \mathrm{b}}$, Jens C. Pruessner ${ }^{\mathrm{c}, \mathrm{e}, \mathrm{g}}$, \\ Sonia J. Lupien ${ }^{\mathrm{a}, \mathrm{b}, \mathrm{h}, *}$
}

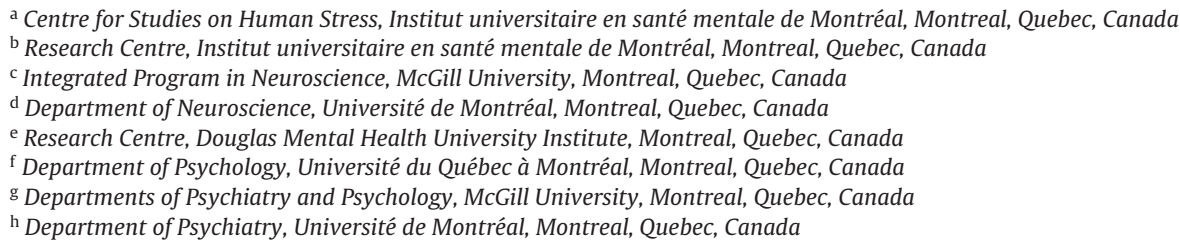

Keywords:

Cortisol

Testosterone

Estradiol

Progesterone

Sex differences

Trier Social Stress Test

\begin{abstract}
A B S T R A C T
Sex differences in stress hormone functions are presumed to depend on sex hormones. And yet, surprisingly few psychoneuroendocrine studies actually assess within-sex variations of testosterone, estradiol, and progesterone when investigating sex-specific activities of the hypothalamic-pituitary-adrenal axis. In this methodological study of 204 healthy adults (60 men), we assessed whether cortisol profiles would differ between the sexes when unadjusted or adjusted for basal sex hormones among both sexes. Reactive cortisol was sampled using 6 saliva samples measured every 10-min as part of the Trier Social Stress Test that generally activates cortisol among men more than women. Diurnal cortisol was sampled over two days at (1) awakening, (2) 30-min thereafter, (3) $1400 \mathrm{~h}$, (4) $1600 \mathrm{~h}$, and (5) bedtime. Sex hormones were collected at baseline before the psychosocial stressor and on two occasions during diurnal cortisol assessment. Repeated-measures analysis of covariance controlled for key covariates in analyses unadjusted or adjusted for sex hormones. Results revealed that men had higher reactive cortisol than women in unadjusted analysis, but this sex difference was attenuated when adjusting for sex hormones. While diurnal cortisol showed no sex differences in unadjusted models, adjusting for sex hormones revealed that women have higher morning cortisol. Correlations using area under the curve formulae revealed intriguing sex-specific associations with progesterone in men and testosterone in women that we propose have implications for social and affective neuroscience. In summary, our results reveal that adjusting for sex hormones alters "sex-specific" reactive and diurnal cortisol profiles.
\end{abstract}

\section{Introduction}

Adrenal and gonadal hormones drive biobehavioral adaptations that ensure survival of organisms and propagation of species. At the epicenter of these evolutionary drives are the hypothalamic-pituitary-adrenal (HPA) axis production of the stress hormone cortisol and the hypothalamic-pituitary-gonadal (HPG) axis production of sex hormones like testosterone, estrogens,

* Corresponding author at: Centre for Studies on Human Stress, 7401 Hochelaga Pavilion Louis Riel, Unit 226, Montreal, Quebec H1N 3M5, Canada.

E-mail address: sonia.lupien@umontreal.ca (S.J. Lupien). and progesterone that have sex-specific functions (Sapolsky, 2004). Until as recently as 1995 , females represented only $17 \%$ of subjects/participants in HPA-axis studies that systematically excluded them due to their cyclic HPG-axis variations (Taylor et al., 2000). Despite increasing inclusion of women today in the psychoneuroedocrine studies of stress physiology, within-sex variations in sex hormones are presumed but rarely actually measured. To address this idiosyncrasy, the current methodological study will assess the extent to which accounting for sex hormones adjust "sex-specific" cortisol profiles.

Sex differences in stress reactive cortisol have a rich history in the stress-disease literature. Over two decades ago, Kirschbaum et al. (1992) reported what appeared to be a strong sex-based differ- 
ence in cortisol stress reactivity. Compared to women, men showed a 2-fold higher cortisol reactivity after public speaking and mental arithmetic in front of an audience (Kirschbaum et al., 1992). In a follow-up study, women using oral contraceptives manifested further attenuation of cortisol stress reactivity (Kirschbaum et al., 1995). Researchers reasoned that this was an estradiol driven effect. Specifically, oral contraceptives contain high levels of estradiol that stimulate the production of cortisol-binding globulin, which in turn removes free cortisol from circulation, therefore resulting in lower net concentrations of active cortisol. Subsequent studies assessed cortisol reactivity at different menstrual cycle phases to further understand HPA-axis and HPG-axis cross-talk. Results revealed that women with low estrogen display reactivity patterns similar to men, while women with high estrogen resemble women using oral contraceptives (Kirschbaum et al., 1999). Thus, acute stimulation of the HPA-axis is related to estrogen regulation in women.

With regards to research on stress reactivity, estrogen and progesterone vary according to the menstrual cycle, hormone-based contraception, and menopause-related hormone therapy later in life (Bouma et al., 2009; Kajantie and Phillips, 2006; Kirschbaum et al., 1999, 1995; Komesaroff et al., 1999; Marinari et al., 1976; Patacchioli et al., 2006; Prinz et al., 2001). Another consideration is that HPA-axis reactivity generally increases with aging, such that stress reactive amplitudes can be three times stronger in postmenopausal women compared to older men (Otte et al., 2005). Whereas estrogen appears to beneficially dampen the HPA-axis in women (Kajantie and Phillips, 2006), the effects of progesterone are usually limited to understanding female reproduction. And yet, both estradiol and progesterone can also modulate neuroendocrine functioning in men (Champagne et al., 2012; Kirschbaum et al., 1996).

Akin to research on "female" hormones, testosterone has been extensively studied in men. Notwithstanding, growing evidence reveals that androgens interact with social contexts among both sexes. Beyond reproductive functions, testosterone interacts with various HPA-axis modulators (e.g., dehydroepiandrosterone antagonizes cortisol) which can contribute to multi-systemic pathophysiology (Juster et al., 2011a). For reasons explained in part by basal differences in HPG-axis functioning, men show the most pronounced differences in HPA-axis responsivity to laboratorybased stressors that elicit social-evaluative threat (Dickerson and Kemeny, 2004; Kajantie and Phillips, 2006; Kirschbaum et al., 1992). Yet for both sexes, enhanced HPG-axis activities like testosterone production are also indicative of challenge and anticipation (Chichinadze and Chichinadze, 2008; Chichinadze et al., 2012a,b), suggesting that the HPG-axis is involved in preparedness to respond for both sexes in stress paradigms.

Complementary to reactive cortisol paradigms, naturalistic variations in HPA-axis functioning can be assessed non-invasively in day-to-day contexts. Upon awakening, the cortisol awakening response (CAR) represents a normal surge in cortisol levels reaching maximal concentrations approximately $30 \mathrm{~min}$ after awakening (Pruessner et al., 1997). This surge is followed by gradually declining cortisol concentrations throughout the day as pulsatile secretion decreases in amplitude and frequency (Clow et al., 2010). The nadir usually occurs around midnight, and cortisol levels start to rise again in the early morning hours (Kudielka et al., 2012; Loucks et al., 2008). Unbeknownst is whether HPA-axis circadian rhythmicity differs between the sexes (Kudielka et al., 2012). Like studies assessing stress reactivity, studies of diurnal cortisol have rarely assessed the functioning of the HPG-axis that may influence inter-individual differences in cortisol profiles.

To address these lacunas, our methodological study explored how sex differences in reactive and diurnal cortisol differ according to general linear models that are (1) unadjusted for sex hormones but otherwise control for key covariates or that are (2) adjusted for testosterone, estradiol, and progesterone sampled among both sexes. Our general hypothesis was that cortisol sex differences in unadjusted models would be statistically attenuated when adjusting for sex hormones. In secondary analyses, we explored the directionality of HPG-axis and HPA-axis associations to further understand cross-talk between these systems by calculating area under the curve scores for key cortisol time-windows in reactive and diurnal conditions. Anticipatory distress was also assessed in relation to HPG-axis and reactive HPA-axis activities.

\section{Methods}

\subsection{Participants}

Participants included 60 men and 144 women of diverse reproductive status (cycling: $n=55$; contraceptive: $n=47$; postmenopausal: $n=42$ ). Participants were recruited from among the employees of the Institut universitaire en santé mentale de Montréal, the largest psychiatric hospital in the Canadian province of Quebec.

Table 1 lists sample information. Inclusion criteria were liberal in order to best represent the health of various professions all from within the same workplace. Women were more likely than men to have a diagnosed mental health condition $(p=.002)$, psychotropic use $(p=.010)$, and past psychiatric sick leave $(p=.007)$. By contrast, men were more likely than women to use illicit drugs $(p=.001)$. Main analyses therefore controlled for illicit drugs and mental health condition that correlated with psychotropic use $(r=.91$, $p<.0001)$ and past psychiatric history $(r=.568, p<.001)$. This heterogeneity in sample characteristics was consistently related to age that we additionally controlled for in main analyses.

\subsection{General protocol}

This study was approved by the local research ethics board of the Institut universitaire en santé mentale de Montréal and adheres to the Declaration of Helsinki.

Testing was conducted by two women (N.D. and A.B.D.) and two men (R.P.J. and O.B.) between October 2011 and December 2012 at the Centre for Studies on Human Stress (Montreal, Quebec, Canada). Access to the participant pool was enabled by hospital administrators who allowed workers to participate during their working hours without prejudice and in full confidentiality. In partnership with our communications department, recruitment was promoted via conferences, intranet advertisements, large banners, face-toface visits to units, and word of mouth. Prospects were instructed to contact our laboratory for a 15 -min screening interview prior to scheduling appointments.

During their first laboratory visit between $13 \mathrm{~h} 00$ and $18 \mathrm{~h} 00$ (arrival time: $M=13 \mathrm{~h} 35, S E=0: 51 \mathrm{~min}$ ) that lasted $90 \mathrm{~min}$, participants (a) were requested to read and sign the consent form; (b) were instructed to provide saliva samples every $10 \mathrm{~min}$ at seven occasions throughout the visit to assess baseline sex hormones and reactive cortisol; (c) completed a cognitive task (data not reported here); (d) were exposed to the Trier Social Stress Test; (e) were instructed in the use of our internet-based questionnaire system; (f) were provided instructions for diurnal salivary collection to assess day-to-day variations in sex hormones and cortisol; and finally (g) were debriefed. Between visits, participants collected saliva samples at home and completed electronic questionnaires that took about $45 \mathrm{~min}$ to finish. During their second visit, participants returned materials and partook in a blood draw and physical examination to assess allostatic load (data not reported here). Participants received 50\$CAD as compensation for their contribution. 
Table 1

Sample characteristics.

\begin{tabular}{|c|c|c|c|}
\hline Information & All & Men & Women \\
\hline $\mathrm{N}$ & 204 & 60 & 144 \\
\hline \multicolumn{4}{|l|}{ Demographics } \\
\hline Age, M (SE) & $40.4(.85)$ & $39.0(1.62)$ & $41.0(1.0$ \\
\hline \multicolumn{4}{|l|}{ Socio-economics } \\
\hline Working hours per week, M (SE) & $34.7(.58)$ & $36.2(1.23)$ & $34.1(.63$ \\
\hline Education, years schooling M (SE) & $16.5(.23)$ & $16.4(.50)$ & $16.5(.25$ \\
\hline Occupational status, $\mathrm{M}(\mathrm{SE})^{\mathrm{a}}$ & $69.4(1.19)$ & $65.9(2.85)$ & $70.8(1.2$ \\
\hline \multicolumn{4}{|l|}{ Civil status } \\
\hline Single, $\%$ & 33.8 & 43.3 & 39.9 \\
\hline In a relationship or common law, $\%$ & 32.8 & 31.7 & 33.3 \\
\hline Married, \% & 22.5 & 18.3 & 24.3 \\
\hline Separated or divorced, \% & 10.8 & 6.7 & 12.5 \\
\hline \multicolumn{4}{|l|}{ Parenthood, number of children } \\
\hline None, $\%$ & 50.0 & 55.0 & 47.9 \\
\hline $1, \%$ & 13.7 & 11.7 & 14.6 \\
\hline $2, \%$ & 27.0 & 21.7 & 29.2 \\
\hline$\leq 3, \%$ & 9.3 & 11.7 & 8.3 \\
\hline Parents with children living at home, \% & 64.3 & 65.7 & 63.7 \\
\hline \multicolumn{4}{|l|}{ Health behaviors } \\
\hline \multicolumn{4}{|l|}{ Caffeinated beverages per day } \\
\hline None, $\%$ & 29.9 & 31.7 & 29.2 \\
\hline $1-2, \%$ & 45.6 & 48.3 & 44.4 \\
\hline$\leq 3, \%$ & 24.5 & 20.0 & 26.4 \\
\hline \multicolumn{4}{|l|}{ Alcohol consumption per week } \\
\hline None, \% & 31.4 & 28.3 & 32.6 \\
\hline $1-5, \%$ & 52.9 & 46.7 & 55.6 \\
\hline$\leq 6, \%$ & 15.7 & 25.0 & 11.8 \\
\hline \multicolumn{4}{|l|}{ Tobacco smoking } \\
\hline Non-smokers, \% & 83.8 & 86.7 & 82.6 \\
\hline Smoker, \% & 16.2 & 13.3 & 17.4 \\
\hline \multicolumn{4}{|l|}{ Elicit drug use } \\
\hline No, \% & 92.6 & 83.3 & 96.5 \\
\hline Yes, \% & 7.4 & 16.7 & 3.5 \\
\hline \multicolumn{4}{|l|}{ Physical and mental health } \\
\hline \multicolumn{4}{|l|}{ Physical health condition(s) } \\
\hline None, \% & 56.4 & 65.0 & 52.8 \\
\hline $1, \%$ & 29.9 & 26.7 & 31.3 \\
\hline$\leq 2, \%$ & 13.7 & 8.3 & 16.0 \\
\hline \multicolumn{4}{|l|}{ Medication(s) prescribed } \\
\hline None, \% & 54.4 & 61.7 & 51.4 \\
\hline $1, \%$ & 31.4 & 26.7 & 33.3 \\
\hline$\leq 2, \%$ & 14.2 & 11.7 & 15.3 \\
\hline \multicolumn{4}{|l|}{ Mental health condition } \\
\hline None, \% & 76.0 & 90.0 & 70.0 \\
\hline Current, \% & 24.0 & 10.0 & 30.0 \\
\hline \multicolumn{4}{|l|}{ Psychotropic(s) prescribed } \\
\hline None, \% & 76.5 & 88.3 & 71.5 \\
\hline 1 or more, $\%$ & 23.5 & 11.7 & 28.5 \\
\hline \multicolumn{4}{|l|}{ Psychiatric history } \\
\hline None, \% & 36.3 & 46.7 & 31.9 \\
\hline Past history, \% & 18.1 & 8.3 & 22.2 \\
\hline Family history, \% & 28.9 & 33.3 & 27.1 \\
\hline Both past and family history, \% & 16.7 & 11.7 & 18.8 \\
\hline Past psychiatric sick leave, \% & 33.8 & 20.0 & 39.6 \\
\hline
\end{tabular}

Abbreviations: $M=$ mean; $S E=$ standard error.

a Occupational status was coded using the Nam-Powers-Boyd method that generates scores based on Canadian census for the median income and education of any given occupational category as previously done (Juster et al., 2013).

\subsection{Anticipatory distress}

The 16-item Primary Appraisal Secondary Appraisal or PASA scale (Gaab et al., 2005) measures anticipatory distress 10-min prior to TSST exposure using a six-point Likert scale. A primary appraisal sub-scale includes "threat" and "challenge" components, while the secondary appraisal sub-scale includes "self-concept of own competence" and "control expectancy" components. Primary and secondary appraisals co-dependently determine an individual's perceptions of threat, which forms the "tertiary appraisal" scale that represents global anticipatory distress. This is calculated as the difference between the primary appraisal and secondary appraisal sub-scales. Original PASA psychometrics revealed sat- isfactory internal consistency ( $\alpha$ s between .63 and .83) and the overall internal consistency for the current sample was similar $(\alpha=.73)$. Given that anticipatory distress predicts between $22 \%$ to $35 \%$ of the variance explaining reactive cortisol (Gaab et al., 2005; Juster et al., 2012), we decided to explore how anticipatory distress is related to HPG-axis functioning and then used it as a covariate in reactive cortisol analyses.

\subsection{Endocrine measures}

\subsubsection{Reactive cortisol}

Participants were exposed to a modified Trier Social Stress Test (Kirschbaum et al., 1993) during their first laboratory visit. Testing was conducted in the afternoon in order to account for circadian variations in endocrine functions. After a 10 -min anticipation period in which participants completed the PASA to assess anticipatory distress and prepared their speeches, they were escorted to a separate room where they delivered a five-minute mock job interview followed by five minutes of mental arithmetic. An unseen confederate on the other side of a one-sided reflective window communicated with participants via an intercommunication device while facing a camera that we deceived participants into thinking was recording.

Research by our group has demonstrated that placing the evaluative audience in this 'Panel-Out' setting maximizes between-sex differences in cortisol reactivity. This modified TSST results in decreased stress reactivity among heterosexual women but not heterosexual men (Juster et al., 2015). In original studies, men exposed to this type of the TSST show no significant differences in HPA axis reactivity compared with the standard performance in front of the audience or original "Panel-In" setting (Andrews et al., 2007). In contrast, heterosexual women exposed to the "Panel-Out" condition show decreased cortisol stress reactivity compared with heterosexual women in the "Panel-In" condition (Wadiwalla et al., 2010).

In total, six saliva samples for cortisol assessment were obtained (1) $10 \mathrm{~min}$ before the TSST, (2) immediately before the TSST, and then (3) $+10,(4)+20,(5)+30$, and ( 6$)+40$ min post-TSST to assess recovery. These time-points were also subsumed using the area under the curve formulae described below.

\subsubsection{Diurnal cortisol}

Over two non-consecutive workdays, unstimulated saliva was collected at five time points: (1) awakening, (2) 30-min after awakening, (3) 14h00 (4) 16h00, and (5) bedtime. Our group has used these time-points to assess diurnal HPA-axis functioning among workers (Juster et al., 2011b; Marchand et al., 2014) and provide sufficient information to establish diurnal curves (Stone et al., 2001). Participants were instructed to avoid eating a major meal, drinking beverages other than water, smoking, chewing gum, or engaging in strenuous physical activity thirty minutes prior to saliva collection. Furthermore, they were asked to refrain from brushing their teeth or flossing two hours before sampling. Upon completion of a sampling day, participants stored samples in their freezer until returning them to our laboratory where they were stored in an industrial freezer at $-20^{\circ} \mathrm{C}$ until assaying when the entire study was completed.

Participants recorded actual sampling times themselves with logbooks and their adherence to scheduled collection time was monitored using the Medication Event Monitoring System (MEMS $^{\mathrm{TM}}$, AARDEX Ltd., Sion, Switzerland). The MEMS cap registers times and dates when the closure is opened with a micro-electronic circuit. Once opened, tubes were retrieved, filled with saliva until $2 \mathrm{ml}$ was attained, and then stored in a plastic bag that was refrigerated. A MEMS Reader USB (Serial number 10959) was used to 
record information when returned to our laboratory and compiled using Powerview software.

MEMS is regarded as the gold standard for monitoring and motivating participant compliance in psychoneuroendocrine field research (Kudielka et al., 2012). MEMS compliance deviation was calculated as the sum absolute difference in minutes for both weekdays between the actual sampling time according to MEMs recordings and the scheduled sampling time for pre-determined time-points (+30 min after awakening, 14h00, and 16h00). Main analyses of diurnal cortisol controlled for mean awakening time $(M=6: 59: 57, S E=0: 05: 12)$ that differed by sex: men woke up later than women $\left(p=.013, \eta^{2}{ }_{p}=.032\right)$. No sex differences were found for MEMS compliance deviation $(p=.769)$. Overall, participants were respectful of assigned time-points, showing only limited deviations in the morning $(M=12.6 \mathrm{~min}, S E=3.05)$ but were less compliant in the afternoon $(M=37.98 \mathrm{~min}, S E=3.05)$. Both AM and PM minutes deviation were summed $(M=49.58 \mathrm{~min}, S E=5.65)$ for use as a covariate in our main analysis.

Diurnal cortisol time-points for the two sampling days were averaged to maximize generalizability. This was justified as reliability among diurnal cortisol time points were acceptable when controlling for MEMS compliance deviation with partial correlations ranging from $r=.43$ to .61 . Among women, diurnal cortisol did not differ according to reproductive status $(p=.832)$.

\subsubsection{Sex hormones}

Salivary testosterone, estradiol, and progesterone concentrations were measured (1) once 10-min before anticipating the TSST and (2) twice during the same days as diurnal cortisol sampling. First, a baseline saliva sample 20-min pre-TSST exposure was collected for all participants in the afternoon $(M=13: 47$, $S E=0: 03 \mathrm{~min}$ ). Second, two additional saliva samples were collected on the same sample days as the diurnal cortisol sampling $(M=14: 19, S E=0: 23 \mathrm{~min})$. Sex-specific reliability analyses revealed acceptable inter-correlations between the two repeated measures of diurnal sex hormones ( $r \mathrm{~s}: .50$ to .83 ) that were therefore averaged. Due to similar sampling times, diurnal sex hormones were correlated with those taken before the TSST: testosterone $(r=.715$, $p<.001)$, estradiol $(r=.322, p<.001)$, and progesterone $(r=.355$, $p<.001)$. It is also noteworthy that correlations among separate sex hormones were generally stronger for men ( $r$ s between .212 and .457) than for women ( $r$ s between .04 and .373).

For women of reproductive age, both pre-TSST and diurnal sex hormones were not associated with days since last menstruation or oral contraceptive use ( $p s>.52$ ). This provides further support for the need to account for within-sex variation in HPG-axis secretory patterns. Indeed, the central argument of our study is that adjusting for sex hormones will alter the presence or absence of "sex-specific" cortisol profiles seen in psychoneuroendocrine studies that rarely measure sex hormones.

\subsubsection{Assay information}

Sterilized $5 \mathrm{ml} 57 \times 15.3 \mathrm{~mm}$ screw cap tubes (Sarstedt ${ }^{\circledR}$, Item No. 62.558.201) were used to collect $2 \mathrm{ml}$ of saliva guided with thick straws. Tubes had $34.9 \times 12.7 \mathrm{~mm}$ cryogenic labels and $11 \mathrm{~mm}$ circular cryogenic labels of different colors to designate different time-points and different hormones so as to help guide participants.

Assays were conducted at the Saliva Laboratory of the Centre for Studies on Human Stress (www.humanstress.ca). Frozen samples were brought to room temperature and centrifuged at $1500 \times g(3000 \mathrm{rpm})$ for $15 \mathrm{~min}$. All assays were run in duplicates and averaged. High-sensitivity enzymeimmuneassays were used for cortisol (Salimetrics ${ }^{\circledR}$, No. 1-3102, sensitivity: 0.012-3 $\mu \mathrm{g} / \mathrm{dl}$ ), estradiol (Salimetrics ${ }^{\circledR}$, No. 1-3702, sensitivity: $1-32 \mathrm{pg} / \mathrm{ml}$ ), and progesterone (Salimetrics ${ }^{\circledR}$, No. 1-1502, sensitivity: $5 \mathrm{pg} / \mathrm{ml}$ ), while testosterone was determined by expanded-range enzymeimmuneassay (Salimetrics ${ }^{\circledR}$, No. $1-2402$, sensitivity: $1 \mathrm{pg} / \mathrm{ml}$ ). Inter-assay and intra-assay coefficients of variance were respectively below $9.27 \%$ and $5.89 \%$ for cortisol, $12.18 \%$ and $3.84 \%$ for testosterone, $10.29 \%$ and $4.23 \%$ for estradiol, and $14.09 \%$ and $6.23 \%$ for progesterone. Assays were duplicated and averaged.

\subsection{Data treatment and statistical analysis}

Statistical analyses used the Statistical Package for the Social Sciences 23 for Macintosh. Missing data for cortisol (reactive: $n=2$; diurnal: $n=16$ ) and and sex hormones (reactive: $n=1$; diurnal: $n=17$ ) are reflected in varying degrees of freedom.

Anticipatory distress was assessed using one-way analysis of variance, Pearson's correlations, and partial correlations controlling for sex. Main analyses used repeated measures analysis of covariance (RM-ANCOVA) for both reactive cortisol and diurnal cortisol with covariates that were unadjusted or adjusted for sex hormones. Default covariates for both reactive cortisol and diurnal cortisol included age, mental health condition, and illicit drugs. Additional covariates for reactive cortisol included anticipatory distress, while diurnal cortisol included awakening time and MEMS compliance deviation. Greenhouse-Geisser corrections are reported whenever appropriate. Post-hoc analyses employed multivariate analysis of covariance split by sex. Effect sizes can be interpreted as a small effect $\left(\eta^{2}{ }_{P} \cong 0.01\right)$, medium effect $\left(\eta^{2}{ }_{P} \cong 0.06\right)$, or large effect $\left(\eta^{2} \cong 0.14\right)$.

Secondary analyses used Pearson's correlations and one-away analysis of variance to explore summary measures of reactive and diurnal contexts. Cortisol data were transformed into timedependent scores: (1) area under the curve with respect to increase (AUCi) and (2) area under the curve with respect to ground (AUCg) formulae (Pruessner et al., 2003). AUCi scores represent increment/decrement sensitivities of the HPA-axis without regard for zero, while AUCg scores represent HPA-axis systemic output starting at zero. Reactive cortisol scores used both AUCi and AUCg, while diurnal cortisol scores used the AUCi to represent the CAR based on the awakening and +30-min samples and the AUCg to represent diurnal systemic output using all samples throughout the day. Recorded time differences for every reactive and diurnal cortisol (e.g., MEMS) sample was used to precisely personalize area under the curve calculations for each participant.

\section{Results}

\subsection{Anticipatory distress}

Analysis of variance revealed that women self-reported higher anticipatory distress than men $\left(p=.021, \eta^{2}{ }_{p}=.028\right)$ before the TSST. This result was driven by primary appraisals related to PASA threat $\left(p=.009, \eta^{2}{ }_{P}=.035\right)$ and challenge $\left(p=.044, \eta^{2}{ }_{P}=.021\right)$ subscales. Partial correlations among the entire sample controlling for sex revealed positive correlations between estradiol concentrations and PASA control expectancy $(r=.157, p=.031)$ as well as PASA threat and systemic cortisol output (AUCg) in the TSST paradigm $(r=.163, p=.024)$. Among men only, sex hormones measured prior to to the TSST were related to PASA components. Testosterone was positively correlated with secondary appraisals $(r=.284, p=.029)$ : this was due to trending secondary appraisals of PASA self-concept of own competence $(r=.228, p=.082)$ and control expectancy $(r=.255, p=.051)$ sub-scales. Progesterone was positively correlated with PASA challenge $(r=.297, p=.024)$ and control expectancy $(r=.295, p=.023)$ subs-scales. 
A1

Unadjusted

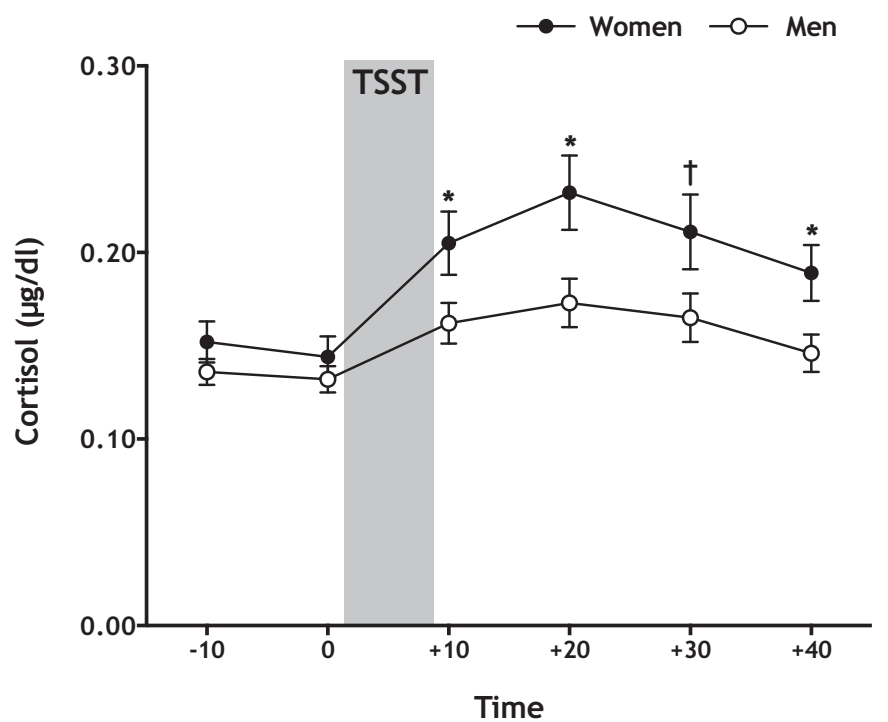

B1

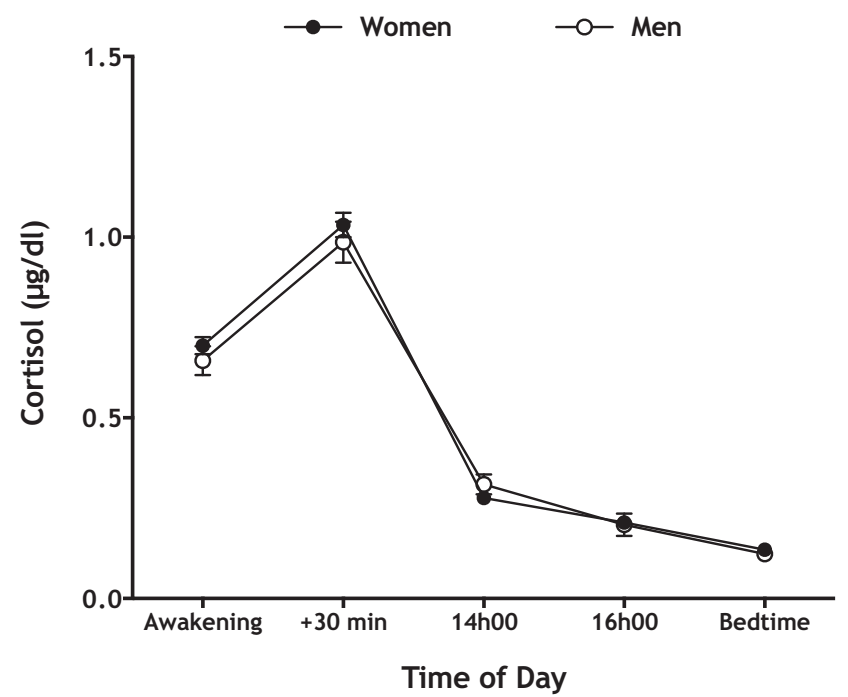

A2 Adjusted

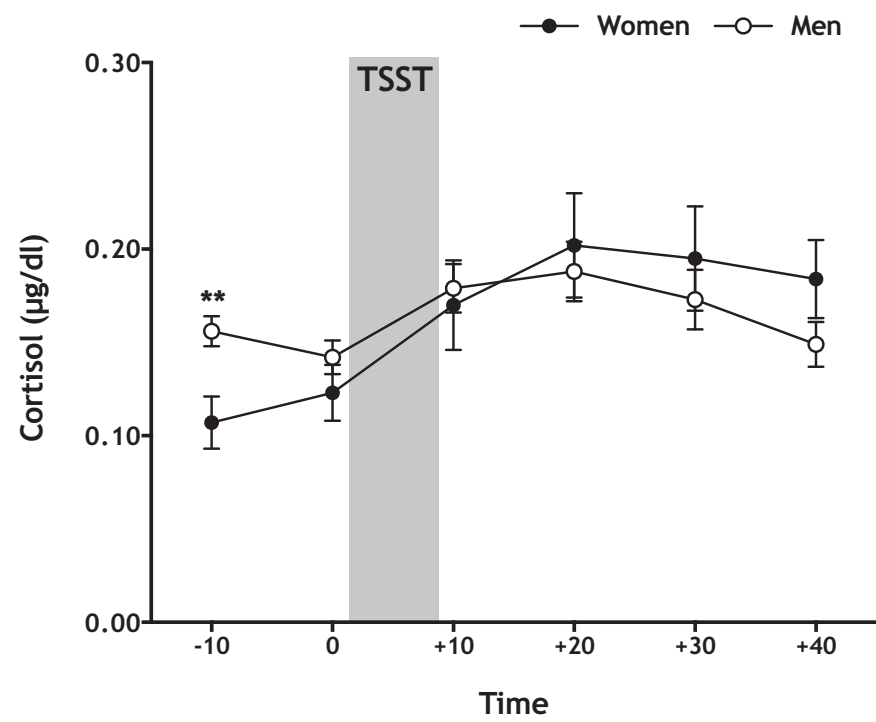

B2

Adjusted

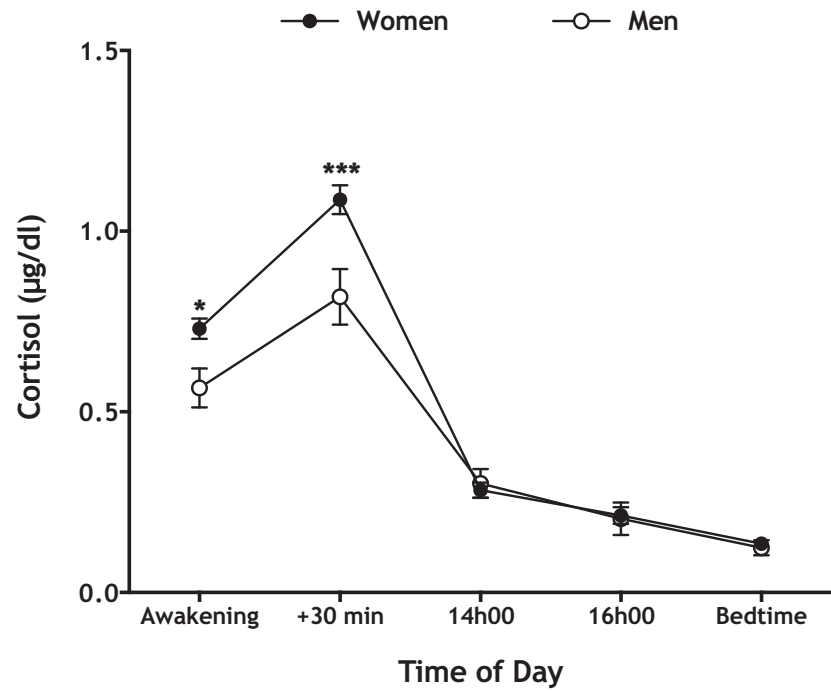

Fig. 1. Estimated means $( \pm$ SE) for reactive cortisol (A1 and A2) and diurnal cortisol (B1 and B2) as a function of sex. Unadjusted models for reactive cortisol controlled for age, mental health condition, illicit drug use, and anticipatory distress (A1). Unadjusted models for diurnal cortisol controlled for age, mental health condition, illicit drug use, awakening time, and compliance deviation (B1). Adjusted models for reactive cortisol (B2) and diurnal cortisol (B2) additionally controlled for testosterone, estradiol, and progesterone.

Note: ${ }^{* *}=p<.01 ;^{*}=p<.05 ; \dagger=p<.10 ;$ TSST $=$ Trier Social Stress Test.

\subsection{Reactive cortisol}

In a RM-ANCOVA unadjusted for sex hormones (Fig. 1A1), we found a significant time effect $\left(F_{(2.41,450.1)}=6.03, p=.001\right.$, $\left.\eta^{2}{ }_{P}=.031\right)$ and between-groups sex difference in which men showed higher cortisol than women $\left(F_{(1,187)}=4.8\right.$, $\left.p=.03, \eta^{2} p=.025\right)$. A trend towards a time $\mathrm{X}$ sex interaction $\left(F_{(2.41,450.1)}=2.45, p=.076, \eta^{2} P=.013\right)$ was due to sex differences $10-\min \left(p=.045, \eta^{2}{ }_{P}=.021\right), 20-\min \left(p=.018, \eta^{2}{ }_{P}=.029\right)$, 30-min $\left(p=.061, \eta^{2}{ }_{P}=.019\right)$, and $40-\min \left(p=.02, \eta^{2}{ }_{P}=.028\right)$ post-TSST. A significant covariation effect emerged for mental health condition $\left(F_{(1,187)}=6.12, p=.014, \eta^{2}=.032\right)$.
In re-analyses adjusting for sex hormones (Fig. 1A2), the sex difference disappeared $(p=.97)$. Instead, we detected a significant time effect $\left(F_{(2.4,434.5)}=7.1, p<.001, \eta^{2}{ }_{P}=.037\right)$ and time $\times$ sex interaction effect $\left(F_{(2.4 .434 .5)}=3.2, p=.035, \eta^{2}{ }_{P}=.017\right)$ : women had higher $C$ than men at baseline $\left(p=.01, \eta^{2}{ }_{P}=.021\right)$, but not thereafter. Covariation effects were detected for time $\mathrm{X}$ testosterone $\left(F_{(2.4,434.5)}=3.1, p=.036, \eta^{2}{ }_{P}=.017\right)$, time $\times$ mental health condition $\left(F_{(2.4,434.5)}=3.0, p=.043, \eta^{2}{ }_{P}=.016\right)$, and mental health condition $\left(F_{(1,181)}=7.03, p=.009, \eta_{P}^{2}=.037\right)$.

Correlations assessed baseline sex hormones in association with reactive cortisol (AUCi) and systemic output (AUCg). For the entire sample, reactive cortisol was negatively associated with 
estradiol ( $r=-.175, p=.014)$, while systemic output was positively correlated with testosterone $(r=.178, p=.011)$. For men, reactive cortisol was negatively correlated only with progesterone $(r=-.256, p=.048)$. For women, reactive cortisol was negatively correlated with testosterone $(r=-.203, p=.016)$ and estradiol $(r=-.176, p=.038)$. When sub-divided according to reproductive status among women, the testosterone effect was driven by menopaused women while the estradiol effect was driven by contraceptive women. No significant associations emerged when assessing cortisol systemic output throughout the TSST session in sex-specific analyses. Men showed marginally higher cortisol systemic output than women $\left(F_{(1,200)}=4.03, p=.046, \eta^{2}{ }_{P}=.020\right)$; however, no sex differences were found for reactive cortisol $\left(p=.431, \eta^{2}{ }_{P}=.003\right)$.

\subsection{Diurnal cortisol}

In a RM-ANCOVA unadjusted for sex hormones (Fig. 1B1), we found a significant time effect $\left(F_{(2.28,410.95)}=23.11, p<.0001\right.$, $\left.\eta^{2}{ }_{P}=.114\right)$. Covariation effects were found for time $\times$ awakening time $\left(F_{(2.28,410.95)}=6.13, p=.001, \eta^{2}{ }_{P}=.033\right)$ and illicit drugs $\left(F_{(1,180)}=8.31, p=.004, \eta^{2}{ }_{P}=.044\right)$. Critically, no sex effects were detected $(p s>.45)$.

In re-analyses adjusting for sex hormones (Fig. 1B2), we found a significant time $\times$ sex interaction effect $\left(F_{(2.37,397.48)}=4.78\right.$, $\left.p=.006, \eta^{2}{ }_{P}=.028\right)$ : compared to men, women had higher cortisol concentrations at awakening $\left(p=.02, \eta^{2}{ }_{p}=.032\right)$ and 30-min thereafter $\left(p=.008, \eta^{2}{ }^{2}=.041\right)$. In between-groups analysis, women showed higher overall cortisol concentrations than men $\left(F_{(1,168)}=4.33, p=.039, \eta^{2}{ }_{P}=.025\right)$. Covariation effects were detected for time $\times$ awakening time $\left(F_{(2.4,397.48)}=5.7\right.$, $\left.p=.002, \eta^{2}{ }_{P}=.033\right)$, time $\times$ testosterone $\left(F_{(2.4,397.48)}=6.8, p=.001\right.$, $\left.\eta^{2}{ }_{P}=.039\right)$, testosterone $\left(F_{(1,168)}=7.1, p=.009, \eta^{2}{ }_{P}=.04\right)$, and illicit drugs $\left(F_{(1,168)}=5.8, p=.017, \eta_{P}^{2}=.033\right)$.

Correlations assessed diurnal sex hormones in association with the CAR (AUCi for awakening and +30 -min) and diurnal systemic output (AUCg for awakening, +30-min, 14h00, 16h00, and bedtime). For the entire sample, diurnal systemic output was positively correlated with testosterone $(r=.163, p=.031)$. For men only, diurnal systemic output was positively correlated with progesterone $(r=.336, p=.016)$ and trending with testosterone $(r=.244, p=.084)$. For women only, diurnal systemic output was positively correlated with testosterone $(r=.231, p=.010)$. When sub-divided according to reproductive status among women, this was driven by cycling women. With the exception of a positive association between the CAR (AUCi) and estradiol among cycling women $(r=.339, p=.020)$, no other associations emerged for the CAR in either sex. No sex differences were found for the CAR $\left(p=.989, \eta^{2}{ }_{P}<.001\right)$ or diurnal systemic output $\left(p=.718, \eta^{2}=.001\right)$.

\section{Discussion}

This study demonstrates that accounting for sex hormones adjusts "sex-specific" reactive and diurnal cortisol profiles. Specifically, covarying for testosterone, estradiol, and progesterone in both sexes attenuated reactive cortisol sex differences while amplifying diurnal cortisol sex differences that might otherwise elude identification. Using summary measures of HPA-axis functioning, we further found sex-specific associations with the HPG-axis. While this has implications for research focusing on sex differences in stress (patho) physiology, accounting for individual difference in sex hormones among men and women may be a prerequisite for studies of complex social behaviors that implicate cross-talk between the HPA-axis and HPG-axis. The following discussion will center on the methodological significance of this study in the context of past and future research.

To the best of our knowledge, our study is the first to link basal HPG-axis functioning to PASA anticipatory distress prior to the TSST. For the entire sample, basal estradiol was correlated with anticipatory distress (PASA control expectancy sub-scale). Unlike past reports (Gaab et al., 2005; Juster et al., 2012), only the PASA threat sub-scale was associated with cortisol systemic output (AUCg). Women self-reported higher overall anticipatory distress than men; however, it is men that showed positive associations among basal testosterone, progesterone, and anticipatory distress, especially PASA sub-scales related to competence and control. Given that women generally self-report greater psychosocial distress than men who are in turn more reactive to the TSST, we can speculate that sex hormones might influence these sex/gender differences (Dedovic et al., 2009).

Sex differences in reactive cortisol were only significant in unadjusted models that were attenuated when adjusting for sex hormones. Furthermore, an interesting observation was that correlations that might otherwise be deemed meaningful for one sex were in fact significant for the opposite sex. For example, testosterone was negatively associated with reactive cortisol (AUCi) most strongly among menopaused women, but not among men. While this association was not strong in magnitude, it does provide novel evidence that testosterone modulates HPA-axis functioning in older women. In a similar vein, hormones generally implicated in female reproduction may modulate cortisol in men. Accordingly, we found that baseline progesterone was negatively associated with reactive cortisol only among men. To assume that HPG-axis and HPA-axis activities vary uniformly between-sex is to ignore a potentially important source of within-sex variation hitherto ignored.

Unsurprisingly for the entire sample combined, testosterone was positively associated with cortisol systemic output (AUCg) throughout the TSST, while estradiol was negatively associated with reactive cortisol (AUCi). The role of estrogen during acute stress is mixed in the stress reactivity literature. There is evidence that estradiol levels rapidly and pervasively increase in response to acute stressors in animal models (Shors et al., 1999) whereby interactions depend on the type, duration, and frequency of the stressor in a multi-phasic manner (Rivier and Rivest, 1991). Moreover, testosterone is converted into estradiol in the brain through aromatization that exerts HPA-axis stimulation (Kudielka et al., 1998). Interestingly, men treated with 48-hours of estradiol show exaggerated HPA-axis responses to the TSST compared to placebo (Kirschbaum et al., 1996). By contrast, estrogens appear to have a stress dampening effect among women (Kajantie and Phillips, 2006; McEwen, 2002). Given the reciprocal interactions among sex and stress hormones, the biological relevance of these findings must be further elucidated with the critical consideration that androgens, estrogens, and progestins may have vastly different effects between the sexes.

Some discussion of broad mechanisms is in order given that HPA-axis and HPG-axis activities are bidirectional (Viau, 2002). Under conditions of chronic stress, the HPA-axis exerts an inhibitory effect on the HPG-axis at the detriment of reproductive behavior and other basal HPG-axis functions. In humans, increased cortisol levels impair the expression of luteinizing hormonereleasing hormone, the secretion of luteinizing hormones, and the synthesis of sex hormones (Rivier and Rivest, 1991; Tilbrook et al., 2000). Conversely, testosterone inhibits the HPA-axis at the hypothalamic level (Terburg et al., 2009), exerting a strong influence on cortisol regulation that may in turn modulate sex differences in stress-related HPA-axis activity (Kerr and Kerr, 2001; Kornstein et al., 2000). In a variety of species, it is males who show lower adrenocorticotropic hormone and cortisol concentrations in 
both basal and stress-induced activity due to inhibitory effects by testosterone (Handa et al., 1994; Young, 1998). And yet in humans, amplified reactive cortisol is typically observed among men when contrasted to women of reproductive age (Dickerson and Kemeny, 2004; Kajantie and Phillips, 2006; Kirschbaum et al., 1992).

In the context of social neuroscience, accounting for HPG-axis activities may be necessary to accurately assess HPA-axis functioning and vice-versa. The dual-hormone hypothesis proposes that basal cortisol moderates testosterone functioning in specific psychosocial contexts that interact with various pro- or anti-social behaviors (Carre and Mehta, 2011; Popma et al., 2007). For example among women athletes, high basal cortisol moderates reduced testosterone reactivity in a competitive event (Edwards and Casto 2015). Conversely among men, low basal cortisol is associated with increased testosterone following exposure to the TSST (Bedgood et al., 2014). Interestingly among both men and women, basal testosterone is positively associated to social threat among individuals with low cortisol (Mehta and Josephs, 2010). It is therefore possible that when cortisol concentrations are high or low, the pathway linking testosterone to behavior is respectively deactivated or activated and this may flip-flop between and within the sexes.

Inter- and intra-individual differences in endocrine functioning are key considerations in the context of specific social contexts. For example following a change in social status like winning a Tetris tournament, basal HPG-axis production of testosterone predicts cortisol reactivity for both sexes (Mehta et al., 2008) and viceversa among men (Zilioli and Watson, 2012). Moreover, enhanced testosterone production in the context of competition can amplify assertiveness and aggression when social status is threatened (Chichinadze et al., 2012a; Chichinadze et al., 2012b). As this is highly likely in the context of the TSST, studying repeated measurement of HPG-axis and HPA-axis products represents an exciting lens from which to further understand within-sex variations in stress physiology that go beyond binary sex differences.

The role of progesterone on the HPA-axis is unclear. Brain imaging research shows that pre-menopausal women with elevated estrogen concentrations show diminished neural responsivity to emotional situations, while progesterone appears to have the opposite enhancing effect (Andreano and Cahill, 2010; Goldstein et al., 2005; Ossewaarde et al., 2011; Protopopescu et al., 2005). This is consistent with animal studies that have revealed progesterone receptors in several key limbic and corticolimbic structures, including the hypothalamus, thalamus, amygdala, hippocampus, and prefrontal cortex (Guerra-Araiza et al., 2002, 2003; Kato et al., 1994). These are the same regions subserving the HPA-axis (Lupien et al., 2009), making co-activation a likely mechanism of action. Yet, little is known about progesterone concentrations in human stress studies, underlining the fact that HPG-axis should be further considered when studying the HPA-axis reactivity.

Despite decades of research, it is also not yet clear if diurnal HPA-axis rhythmicity differs between the sexes (Clow et al., 2010; Kudielka et al., 2012; Wolfram et al., 2011). Like reactive cortisol studies, it is rather striking that sex hormones are rarely actually measured, but instead assumed to vary uniformly among groups according to reproductive status that is only intermittingly related to diurnal cortisol (Kudielka and Kirschbaum, 2003; Pruessner et al., 1997). This was not the case in the current study. Instead, we found that women showed higher cortisol concentrations at awakening and +30-min thereafter in comparison to men in adjusted but not unadjusted models. Similar to analyses of reactive cortisol, testosterone was associated with diurnal cortisol systemic output for the entire sample, but only for women in sex-stratified analyses.

HPG-axis confounding of the HPA-axis has generally only focused on estradiol or progesterone variation among women (Kirschbaum et al., 1999) without consideration that testosterone may be of functional significance. By contrast, diurnal cortisol systemic output was associated with progesterone in men, consistent with research showing that progesterone is biological meaningful among men (Champagne et al., 2012). This may represent overall concentration differences between men and women as well as sensitization differences to bound and free fractions of sex hormones. Taken together, an important take home message of our study is that sex hormones provide a means to conceptualize sex continuously rather than categorically, therefore allowing psychoneuroendocrine researchers to reconceptualize "sex-specific" phenomena.

\subsection{Strengths and limitations}

Several considerations strengthen and weaken our findings. Despite a respectable sample size, men were under-represented. On the other hand, women of various reproductive statuses (e.g., cycling, contraceptive, menopaused) were well represented, even though they did not significantly differ from each other for key study variables. Our sample was also quite heterogeneous since individuals reported varying degrees of mental health despite still being healthy and working. This ultimately limits comparison to other studies using stricter exclusion criteria and also lacks generalizability given the common hospital workplace. Nevertheless, our rigorous intra-individual assessment of HPA-axis functioning provided us with a rich contextual window from which to observe individual differences. To improve this approach further, future studies could assess multiple measures of sex hormones to understand dynamic functioning that we could not address with our limited measurement of basal sex hormones constrained to afternoon sampling.

Lifelong dynamics and exposure to sex steroids differ greatly between the sexes. Men experience age-related linear declines in testosterone levels whereas women's lives are characterized by constant periodic fluctuations including menarche, menstruation, pregnancies, and menopause. Consequently, biological sex differences observed might depend on sex hormones (i.e., androgens versus estrogens), categorized concentrations of sex hormones (i.e., phase of the menstrual cycle, pregnancy, menopause), and lifelong exposures to changes in sex hormones (i.e., age at puberty, number of pregnancies, exogenous hormone administration (Juster et al., 2011a). As lifelong differences in the hormonal milieu vary greatly among individuals (Lord et al., 2009), this study provides further evidence that dichotomizations according to sex are oversimplified. Notwithstanding, we acknowledge that it is not always feasible to assess a lifetime of factors nor systematically include sex hormones in every study assessing the HPA-axis. Technological advances in biochemical quantification (e.g., liquid chromatography) of multiple steroid hormones could eventually help limit the costs associated with taking more expensive approaches to assessing neuroendocrine profiles (Gao et al., 2015).

Measuring sex as a binary "male/female" classification is not sufficient when scientists are attempting to understand differences between men, women, and gender diverse individuals (Wainer and Wainer, 2012). Adjusting for sex hormones allows us to robustly account for biological sex-based modulation of HPA-axis activities, thus allowing researchers to thereafter sift out the effects of socio-cultural gender (Juster et al., 2015). Notwithstanding, our study is a cross-sectional snapshot that did not account for organizational and activational processes related to the HPG-axis throughout life. Furthermore, chromosomal-dependent sex differences (Kuljis et al., 2013) represent another layer of genetic complexity. Indeed, various other factors related to biological constitution (e.g., organs, metabolism) represent additional considerations for future research aimed at unravelling the mechanisms at play. Lastly, we believe that transgender individuals represents an 
understudied population (Institute of Medicine, 2011) that ought to be included to understand how transitioning and synthetic sex hormones correlate with cortisol profiles.

\section{Conclusions}

In summary, we argue that accounting for sex hormone variations in both sexes may be the best way to adjust for biological sex-based modulation of stress physiology. This could help eliminate over-reliance on self-reports (Bachand et al., 2009; Jukic et al., 2008; Small et al., 2007) that do not consistently correspond with endocrine concentrations presumed to vary as a function of reproductive statuses (e.g., contraceptive, luteal/follicular women, menopause/andropause). Beyond this methodological consideration, our findings suggest that within-sex variations in sex hormone concentrations can help explain additional variance in reactive and diurnal cortisol profiles. Indeed, perhaps the most intriguing finding was that sex-specific associations among cortisol profiles were observed with progesterone in men and testosterone in women. Given vast inter- and intra-individual differences in basal steroid activities, measuring sex hormones is essential when investigating stress physiology in the aim of teasing apart "sex-specific" disease vulnerabilities.

\section{Conflict of interest}

The authors unanimously declare no conflicts of interest.

\section{Role of the funding source}

This project was supported by the Canadian Institutes of Health Research (CIHR) Institute of Gender and Health (Grant No. 222055) awarded to S.J. Lupien (Principal Investigator), E. Kouassi, and A. Lesage. S.J. Lupien held a senior investigator chair on Gender and Mental Health from the CIHR Institute of Gender and Health (Grant No. GSC 91039). R.P. Juster held a doctoral scholarship from the CIHR Institute of Aging (Grant No. SIA 95402) and support from the Research Team on Work and Mental Health (ERTSM).

\section{Contributors}

R.P. Juster and S.J. Lupien contributed to the study design. Testing and data collection was performed by R.P. Juster, N. Durand, A.B. Desrochers, and O. Bourdon. Logistic support was provided by C. Raymond and N. Wan. R.P. Juster analyzed and interpreted the data under the supervision of J.C. Pruessner and S.J. Lupien. R.P. Juster drafted the manuscript and all co-authors approved the final version of the manuscript for submission.

\section{Acknowledgements}

We wish to thank our participants for their contributions. This project would not have been possible without the hard work of our technician Helen Findlay. Thanks are also expressed to Joanne Beausejour for administrative assistance and Catherine Dion for communications support. We also thank Brigitte Kudielka and Victor Viau for scientific advice as well as Gillian Einstein and Mark Ellenbogen for their academic support.

\section{References}

Andreano, J.M., Cahill, L., 2010. Menstrual cycle modulation of medial temporal activity evoked by negative emotion. Neuroimage 53, 1286-1293.

Andrews, J., Wadiwalla, M., Juster, R.P., Lord, C., Lupien, S.J., Pruessner, J.C., 2007. Effects of manipulating the amount of social-evaluative threat on the cortisol stress response in young healthy men. Behav. Neurosci. 121, 871-876.
Bachand, A.M., Cragin, L.A., Reif, J.S., 2009. Reliability of retrospectively assessed categorical menstrual cycle length data. Ann. Epidemiol. 19, 501-503.

Bedgood, D., Boggiano, M.M., Turan, B., 2014. Testosterone and social evaluative stress: the moderating role of basal cortisol. Psychoneuroendocrinology 47, 107-115.

Bouma, E.M., Riese, H., Ormel, J., Verhulst, F.C., Oldehinkel, A.J., 2009. Adolescents' cortisol responses to awakening and social stress; effects of gender, menstrual phase and oral contraceptives. The TRAILS study. Psychoneuroendocrinology 34, 884-893.

Carre, J.M., Mehta, P.H., 2011. Importance of considering testosterone-cortisol interactions in predicting human aggression and dominance. Aggress. Behav. 37, 489-491

Champagne, J., Lakis, N., Bourque, J., Stip, E., Lipp, O., Mendrek, A., 2012. Progesterone and cerebral function during emotion processing in men and women with schizophrenia. Schizophr. Res. Treatment 2012, 917901.

Chichinadze, K., Chichinadze, N., 2008. Stress-induced increase of testosterone: contributions of social status and sympathetic reactivity. Physiol. Behav. 94, 595-603.

Chichinadze, K., Chichinadze, N., Gachechiladze, L., Lazarashvili, A., 2012a. The role of androgens in regulating emotional state and aggressive behavior. Rev. Neurosci. 23, 123-133.

Chichinadze, K., Lazarashvili, A., Chichinadze, N., Gachechiladze, L., 2012b. Testosterone dynamics during encounter: role of emotional factors. J. Comp. Physiol. A Neuroethol. Sens. Neural Behav. Physiol. 198, 485-494.

Clow, A., Hucklebridge, F., Stalder, T., Evans, P., Thorn, L., 2010. The cortisol awakening response: more than a measure of HPA axis function. Neurosci. Biobehav. Rev. 35, 97-103.

Dedovic, K., Wadiwalla, M., Engert, V., Pruessner, J.C., 2009. The role of sex and gender socialization in stress reactivity. Dev. Psychol. 45, 45-55.

Dickerson, S.S., Kemeny, M.E., 2004. Acute stressors and cortisol responses: a theoretical integration and synthesis of laboratory research. Psychol. Bull 130 355-391.

Edwards, D.A., Casto, K.V., 2015. Baseline cortisol moderates testosterone reactivity to women's intercollegiate athletic competition. Physiol. Behav. 142, 48-51.

Gaab, J., Rohleder, N., Nater, U.M., Ehlert, U., 2005. Psychological determinants of the cortisol stress response: the role of anticipatory cognitive appraisal. Psychoneuroendocrinology 30, 599-610.

Gao, W., Stalder, T., Kirschbaum, C., 2015. Quantitative analysis of estradiol and six other steroid hormones in human saliva using a high throughput liquid chromatography-tandem mass spectrometry assay. Talanta 143, 353-358.

Goldstein, J.M., Jerram, M., Poldrack, R., Ahern, T., Kennedy, D.N., Seidman, L.J., Makris, N., 2005. Hormonal cycle modulates arousal circuitry in women using functional magnetic resonance imaging. J. Neurosci. 25, 9309-9316.

Guerra-Araiza, C., Coyoy-Salgado, A., Camacho-Arroyo, I., 2002. Sex differences in the regulation of progesterone receptor isoforms expression in the rat brain. Brain Res. Bull. 59, 105-109.

Guerra-Araiza, C., Villamar-Cruz, O., Gonzalez-Arenas, A., Chavira, R., Camacho-Arroyo, I., 2003. Changes in progesterone receptor isoforms content in the rat brain during the oestrous cycle and after oestradiol and progesterone treatments. J. Neuroendocrinol. 15, 984-990.

Handa, R.J., Burgess, L.H., Kerr, J.E., O’Keefe, J.A., 1994. Gonadal steroid hormone receptors and sex differences in the hypothalamo-pituitary-adrenal axis. Horm. Behav. 28 464-476.

Institute of Medicine, 2011. The Health of Lesbian, Gay, Bisexual, and Transgender People: Building a Foundation for Better Understanding. The National Academies Press, Washington, DC

Jukic, A.M., Weinberg, C.R., Wilcox, A.J., McConnaughey, D.R., Hornsby, P., Baird, D.D., 2008. Accuracy of reporting of menstrual cycle length. Am. J. Epidemiol. 167, 25-33.

Juster, R.P., Bizik, G., Picard, M., Arsenault-Lapierre, G., Sindi, S., Trepanier, L. Marin, M.F., Wan, N., Sekerovic, Z., Lord, C., Fiocco, A.J., Plusquellec, P., McEwen, B.S., Lupien, S.J., 2011a. A transdisciplinary perspective of chronic stress in relation to psychopathology throughout life span development. Dev. Psychopathol. 23, 725-776.

Juster, R.P., Sindi, S., Marin, M.F., Perna, A., Hashemi, A., Pruessner, J.C., Lupien, S.J. 2011b. A clinical allostatic load index is associated with burnout symptoms and hypocortisolemic profiles in healthy workers. Psychoneuroendocrinology 36, 797-805

Juster, R.P., Perna, A., Marin, M.F., Sindi, S., Lupien, S.J., 2012. Timing is everything: anticipatory stress dynamics among cortisol and blood pressure reactivity and recovery in healthy adults. Stress $15,569-577$.

Juster, R.P., Moskowitz, D.S., Lavoie, J., D’Antono, B., 2013. Sex-specific interaction effects of age, occupational status, and workplace stress on psychiatric symptoms and allostatic load among healthy Montreal workers. Stress 16, 616-629.

Juster, R.P., Hatzenbuehler, M.L., Mendrek, A., Pfaus, J.G., Smith, N.G., Johnson, P.J., Lefebvre-Louis, J.P., Raymond, C., Marin, M.F., Sindi, S., Lupien, S.J., Pruessner, J.C., 2015. Sexual orientation modulates endocrine stress reactivity. Biol. Psychiatry 77, 668-676.

Kajantie, E., Phillips, D.I., 2006. The effects of sex and hormonal status on the physiological response to acute psychosocial stress. Psychoneuroendocrinology 31, 151-178.

Kato, J., Hirata, S., Nozawa, A., Yamada-Mouri, N., 1994. Gene expression of progesterone receptor isoforms in the rat brain. Horm. Behav. 28, 454-463. 
Kerr, L.K., Kerr Jr., L.D., 2001. Screening tools for depression in primary care: the effects of culturegender, and somatic symptoms on the detection of depression. West. J. Med. 175, 349-352.

Kirschbaum, C., Wust, S., Hellhammer, D., 1992. Consistent sex differences in cortisol responses to psychological stress. Psychosom. Med. 54, 648-657.

Kirschbaum, C., Pirke, K.M., Hellhammer, D.H., 1993. The 'Trier Social Stress Test'-a tool for investigating psychobiological stress responses in a laboratory setting. Neuropsychobiology 28, 76-81.

Kirschbaum, C., Pirke, K.M., Hellhammer, D.H., 1995. Preliminary evidence for reduced cortisol responsivity to psychological stress in women using oral contraceptive medication. Psychoneuroendocrinology 20, 509-514.

Kirschbaum, C., Schommer, N., Federenko, I., Gaab, J., Neumann, O., Oellers, M. Rohleder, N., Untiedt, A., Hanker, J., Pirke, K.M., Hellhammer, D.H., 1996. Short-term estradiol treatment enhances pituitary-adrenal axis and sympathetic responses to psychosocial stress in healthy young men. J. Clin. Endocrinol. Metab. 81, 3639-3643.

Kirschbaum, C., Kudielka, B.M., Gaab, J., Schommer, N.C., Hellhammer, D.H., 1999. Impact of gendermenstrual cycle phase, and oral contraceptives on the activity of the hypothalamus-pituitary-adrenal axis. Psychosom. Med. 61, 154-162.

Komesaroff, P.A., Esler, M.D., Sudhir, K., 1999. Estrogen supplementation attenuates glucocorticoid and catecholamine responses to mental stress in perimenopausal women. J. Clin. Endocrinol. Metab. 84, 606-610.

Kornstein, S.G., Schatzberg, A.F., Thase, M.E., Yonkers, K.A., McCullough, J.P., Keitner, G.I., Gelenberg, A.J., Ryan, C.E., Hess, A.L., Harrison, W., Davis, S.M. Keller, M.B., 2000. Gender differences in chronic major and double depression. J. Affect. Disord. 60, 1-11.

Kudielka, B.M., Kirschbaum, C., 2003. Awakening cortisol responses are influenced by health status and awakening time but not by menstrual cycle phase. Psychoneuroendocrinology 28, 35-47.

Kudielka, B.M., Hellhammer, J., Hellhammer, D.H., Wolf, O.T., Pirke, K.M., Varadi, E., Pilz, J., Kirschbaum, C., 1998. Sex differences in endocrine and psychological responses to psychosocial stress in healthy elderly subjects and the impact of a 2-week dehydroepiandrosterone treatment. J. Clin. Endocrinol. Metab. 83, 1756-1761.

Kudielka, B.M., Gierens, A., Hellhammer, D.H., Wust, S., Schlotz, W., 2012. Salivary cortisol in ambulatory assessment-some dossome don'ts and some open questions. Psychosom. Med. 74, 418-431.

Kuljis, D.A., Loh, D.H., Truong, D., Vosko, A.M., Ong, M.L., McClusky, R., Arnold, A.P. Colwell, C.S., 2013. Gonadal- and sex-chromosome-dependent sex differences in the circadian system. Endocrinology 154, 1501-1512.

Lord, C., Duchesne, A., Pruessner, J.C., Lupien, S.J., 2009. Measuring indices of lifelong estrogen exposure: self-report reliability. Climacteric 12, 387-394.

Loucks, E., Juster, R.P., Pruessner, J.C., 2008. Neuroendocrine biomarkers, allostatic load, and the challenge of measurement: a commentary on gersten. Soc. Sci. Med. 66, 525-530.

Lupien, S.J., McEwen, B.S., Gunnar, M.R., Heim, C., 2009. Effects of stress throughout the lifespan on the brain: behaviour and cognition. Nat. Rev. Neurosci. 10, 434-445.

Marchand, A., Juster, R.P., Durand, P., Lupien, S.J., 2014. Burnout symptom sub-types and cortisol profiles: what's burning most? Psychoneuroendocrinology 40, 27-36.

Marinari, K.T., Leshner, A.I., Doyle, M.P., 1976. Menstrual cycle status and adrenocortical reactivity to psychological stress. Psychoneuroendocrinology 1 , 213-218.

McEwen, B., 2002. Estrogen actions throughout the brain. Recent Prog. Horm. Res. 57, 357-384.

Mehta, P.H., Josephs, R.A., 2010. Testosterone and cortisol jointly regulate dominance: evidence for a dual-hormone hypothesis. Horm. Behav. 58, 898-906.

Mehta, P.H., Jones, A.C., Josephs, R.A., 2008. The social endocrinology of dominance: basal testosterone predicts cortisol changes and behavior following victory and defeat. J. Pers. Soc. Psychol. 94, 1078-1093.

Ossewaarde, L., Qin, S., Van Marle, H.J., van Wingen, G.A., Fernandez, G., Hermans, E.J., 2011. Stress-induced reduction in reward-related prefrontal cortex function. Neuroimage 55, 345-352.
Otte C. Hart S, Neylan, T.C. Marmar, C.R., Yaffe, K, Mohr, D.C., 2005. A meta-analysis of cortisol response to challenge in human aging: importance of gender. Psychoneuroendocrinology 30, 80-91.

Patacchioli, F.R., Simeoni, S., Monnazzi, P., Pace, M., Capri, O., Perrone, G., 2006. Menopause, mild psychological stress and salivary cortisol: influence of long-term hormone replacement therapy (HRT). Maturitas 55, 150-155.

Popma, A., Vermeiren, R., Geluk, C.A., Rinne, T., van den Brink, W., Knol, D.L., Jansen, L.M., van Engeland, H., Doreleijers, T.A., 2007. Cortisol moderates the relationship between testosterone and aggression in delinquent male adolescents. Biol. Psychiatry 61, 405-411.

Prinz, P., Bailey, S., Moe, K., Wilkinson, C., Scanlan, J., 2001. Urinary free cortisol and sleep under baseline and stressed conditions in healthy senior women: effects of estrogen replacement therapy. J. Sleep Res. 10, 19-26.

Protopopescu, X., Pan, H., Altemus, M., Tuescher, O., Polanecsky, M., McEwen, B. Silbersweig, D., Stern, E., 2005. Orbitofrontal cortex activity related to emotional processing changes across the menstrual cycle. Proc. Natl. Acad. Sci. U. S. A. 102, 16060-16065.

Pruessner, J.C. Wolf, O.T. Hellhammer, D.H., Buske-Kirschbaum, A., von Auer, K. Jobst, S., Kaspers, F., Kirschbaum, C., 1997. Free cortisol levels after awakening: a reliable biological marker for the assessment of adrenocortical activity. Life Sci. 61, 2539-2549.

Pruessner, J.C., Kirschbaum, C., Meinlschmid, G., Hellhammer, D.H., 2003. Two formulas for computation of the area under the curve represent measures of total hormone concentration versus time-dependent change. Psychoneuroendocrinology 28, 916-931.

Rivier, C., Rivest, S., 1991. Effect of stress on the activity of the hypothalamic-pituitary-gonadal axis: peripheral and central mechanisms. Biol. Reprod. 45, 523-532.

Sapolsky, R.M., 2004. Why Zebras Don't Get Ulcers: The Acclaimed Guide to Stress, Stress Related Disease, and Coping. Henry Holt and Company, New York.

Shors, T.J., Pickett, J., Wood, G., Paczynski, M., 1999. Acute stress persistently enhances estrogen levels in the female rat. Stress 3, 163-171.

Small, C.M., Manatunga, A.K., Marcus, M., 2007. Validity of self-reported menstrual cycle length. Ann. Epidemiol. 17, 163-170.

Stone, A.A., Schwartz, J.E., Smyth, J., Kirschbaum, C., Cohen, S., Hellhammer, D. Grossman, S., 2001. Individual differences in the diurnal cycle of salivary free cortisol: a replication of flattened cycles for some individuals. Psychoneuroendocrinology 26, 295-306.

Taylor, S.E., Klein, L.C., Lewis, B.P., Gruenewald, T.L., Gurung, R.A., Updegraff, J.A., 2000. Biobehavioral responses to stress in females: tend-and-befriend, not fight-or-flight. Psychol. Rev. 107, 411-429.

Terburg, D., Morgan, B., van Honk, J., 2009. The testosterone-cortisol ratio: a hormonal marker for proneness to social aggression. Int. J. Law Psychiatry 32, 216-223.

Tilbrook, A.J., Turner, A.I., Clarke, I.J., 2000. Effects of stress on reproduction in non-rodent mammals: the role of glucocorticoids and sex differences. Rev. Reprod. 5, 105-113.

Viau, V., 2002. Functional cross-talk between the hypothalamic-pituitary-gonadal and -adrenal axes. J. Neuroendocrinol. 14, 506-513.

Wadiwalla, M., Andrews, J., Lai, B., Buss, C., Lupien, S.J., Pruessner, J.C., 2010. Effects of manipulating the amount of social-evaluative threat on the cortisol stress response in young healthy women. Stress 13, 214-220.

Wainer, J., Wainer, Z., 2012. Gender matters. In: Schenck-Gustafsson, K., DeCola, P.R., Pfaff, D.W., Pisetsky, D.S. (Eds.), Handbook of Clinical Gender Medicine. Karger AG, Basel, pp. 1-4.

Wolfram, M., Bellingrath, S., Kudielka, B.M., 2011. The cortisol awakening response (CAR) across the female menstrual cycle. Psychoneuroendocrinology 36, 905-912.

Young, E.A., 1998. Sex differences and the HPA axis: implications for psychiatric disease. J. Gend. Specif. Med. 1, 21-27.

Zilioli, S., Watson, N.V., 2012. The hidden dimensions of the competition effect: basal cortisol and basal testosterone jointly predict changes in salivary testosterone after social victory in men. Psychoneuroendocrinology 37, 1855-1865. 